\title{
Satisfacción en el trabajo y en la profesión enfermera
}

M. Rosa Girbau Garcia ${ }^{a}$, Jordi Galimany Masclans ${ }^{b}$ y Eva Garrido Aguilar ${ }^{c}$

a Enfermera y Doctora en Ciencias Enfermeras, Profesora Titular de la Escuela Universitaria de Enfermería de la Universidad de Barcelona, Barcelona, España

b Enfermero y Master en Liderazgo y Gestión de Servicios, Profesor Colaborador Permanente de la Escuela Universitaria de Enfermería de la Universidad de Barcelona, Barcelona, España

c Profesora Colaboradora de la Escuela Universitaria de Enfermería de la Universidad de Barcelona, Barcelona, España

\section{Introducción}

La satisfacción se considera como un constructo de carácter multidimensional y asociada a la calidad de vida en términos de condiciones objetivas y componentes subjetivos ${ }^{1}$, siendo inevitable la relación satisfacción laboral y conducta o acción.

El análisis de la satisfacción en el trabajo (ST), como indicador del equilibrio entre trabajo y vida familiar, se entiende como el grado de bienestar percibido por el individuo, siendo un valor en alza en todas las organizaciones. Se incluye en cualquier evaluación conductas de liderazgo y compromiso con la organización ${ }^{6}$. Sin embargo, resulta paradójico que, a pesar del incremento de estudios de la satisfacción o insatisfacción en el trabajo, los avances conseguidos en la práctica sean poco significativos, ya que se detectan las posibles causas pero posiblemente no se aplican las soluciones.

\section{Factores que inciden en} la satisfacción en el trabajo En la actualidad también se considera que la satisfacción de los profesionales de enfermería está relacionada con las condiciones de trabajo, las relaciones,

\section{El análisis de la satisfacción en el trabajo (ST), como indicador}

del equilibrio entre trabajo y vida familiar, se entiende como el grado de bienestar percibido por el individuo, siendo un valor en alza en todas las organizaciones.

de la calidad global de los servicios de salud, ya que la excelencia de los cuidados prestados a la población en los centros asistenciales está vinculada en gran medida a la satisfacción de sus profesionales ${ }^{2}$.

Según Peya ${ }^{3}$ y otros autores ${ }^{4,5}$, es una de las variables de estudio más frecuentes en investigaciones relativas a los fenómenos relacionados con las teorías organizativas y los puestos de trabajo en la profesión enfermera y otras. También son muchos los estudios sobre ST y el reconocimiento profesional, las expectativas, la autonomía laboral, la posibilidad de promoción, así como el liderazgo, las políticas de flexibilidad horaria y los protocolos organizativos, pudiendo desencadenar reacciones afectivas y cognitivas que condicionarán la actitud de las enfermeras hacia el entorno laboral ${ }^{7-9}$.

En un análisis presentado por Lu et al. ${ }^{10}$ sobre ST entre las enfermeras se dice que, a pesar de ser una variable de estudio frecuente en investigaciones sobre conducta organizacional, se precisan más estudios que incorporen variables que incidan en la satisfacción, como los aspectos personales, profesionales y ambientales, para entender la importancia de la multiplicidad de factores que pueden influir en la satisfacción ${ }^{11}$.

En un estudio multicéntrico realizado en diferentes países ${ }^{12}$ se muestra que el indice de insatisfacción laboral de las enfermeras se sitúa entre el $17 \%$ de las alemanas, 36\% de las inglesas, 33\% de las canadienses y el $41 \%$ de las enfermeras en Estados Unidos. En cambio, otro estudio realizado con enfermeras americanas $^{13}$ argumenta que las variables demográficas tienen una gran influencia en las dimensiones de la ST, siendo a su vez predictoras de la calidad de vida.

Sin embargo, en una amplia revisión de los estudios realizados con enfermeras comunitarias americanas ${ }^{14}$ para analizar la ST de éstas, se manifestó la dificultad de sacar conclusiones globales como consecuencia de las notables diferencias en la metodología, escenarios y características de la muestra. Añadían los autores que las políticas de la organización, las condiciones de trabajo, el contacto con los pacientes y las relaciones con los compañeros eran variables constantes en los diferentes estudios revisados, con una influencia directa con la satisfacción.

Una investigación con enfermeras de Escocia y Noruega ${ }^{15}$ concluyó que la satisfacción estaba relacionada con 
factores laborales

edición española

la opinión que tenía la población de la calidad de los cuidados ofrecidos por estas profesionales, así como la relación con sus compañeros, aspecto éste que se confirmó en otro estudio realizado con enfermeras mexicanas ${ }^{16}$, en el que se consideraba que la variable satisfacción de las enfermeras y del usuario tenía una alta correlación.

Algunos autores, como Andersen ${ }^{17}$ y Hjortdahl ${ }^{18}$, establecen una relación positiva y lineal entre la satisfacción de los profesionales de enfermería, la calidad de los cuidados prestados y la utilización de los servicios de Atención Primaria de la Salud (APS) por parte de la población, y consideran: factores de necesidad, a partir de la salud percibida por el paciente, acompañada de morbilidad objetiva; factores predisponentes, como la edad, la cronicidad, etc., y factores facilitadores, como son las características de la organización y la implantación o no de programas preventivos.

En el ámbito de la APS en España, se presentó un estudio realizado en Madrid en el año 2000 para conocer, entre otros, la ST percibida por los profesionales sanitarios ${ }^{19}$, obteniendo un nivel de satisfacción en enfermería de 3,24 sobre 10, siendo la promoción profesional y la tensión laboral los ítems peor valorados. Destacamos que en ese lugar se realizó otro estudio posterior en el año $2003^{20}$, en el que se medía la satisfacción global como indicador de la calidad de vida, con un resultado mejor para el conjunto de trabajadores de la APS, siendo de 6,15 sobre 10 en las enfermeras. Al mismo tiempo, los resultados de otro estudio realizado en nuestro país en el año $2004^{21}$ confirman que el nivel de satisfacción global de las enfermeras es medio, con un 3,9 sobre 7, y la retribución y la carga de trabajo son los ítems con menor valoración.

Por otro lado, algunos autores 22 plantean la existencia de una relación significativa entre la percepción de las demandas emocionales asociadas al rol familiar y la ST de los profesionales de atención primaria, considerando éstos organización y que la distinguen de otras organizaciones. Estos atributos son de permanencia relativa en el tiempo e influyen en la conducta de las personas que forman parte de ella.

Según Caballero ${ }^{24}$, Hoppoch publicó ya en el año 1935 del siglo pasado una monografía sobre el clima laboral y su impacto en la satisfacción de los profesionales. En ella enfatizaba sobre la multiplicidad de factores que influyen sobre las actitudes de éstos, incluyendo aspectos fisiológicos y psicológicos, a la vez que oportunidades de desarrollo, estatus y reconocimiento, e intentaba

\section{Resulta paradójico que, a pesar del incremento de estudios de} la satisfacción o insatisfacción en el trabajo, los avances conseguidos en la práctica sean poco significativos, ya que se detectan las posibles causas pero posiblemente no se aplican las soluciones.

que existen diferencias notables en los resultados, según el centro, el equipo, el tipo de organización y el ambiente laboral.

El clima organizacional y laboral puede favorecer el buen funcionamiento del servicio asistencial y actuar como catalizador en la ST de sus profesionales, ya que las personas se pueden considerar como el principal activo de cualquier organización ${ }^{23}$, entendido como el conjunto de atributos o características que definen objetivamente una explicar qué necesidades o valores debían ser satisfechos por el individuo dentro de una organización.

En la actualidad algunos autores $25-27$ proponen que la relación entre ST y clima organizacional debería revisarse, sobre todo en profesiones de servicios sanitarios, ya que si la ST es positiva, facilita el compromiso y la fidelización con la empresa, lo que resulta beneficioso para ésta, con mayor efectividad y con la mejora de la calidad asistencial y de la relación entre los miembros del equipo de salud. 
Por otro lado, diferentes estudios ${ }^{28-29}$ evidencian que algunas de las dimensiones más valoradas por los profesionales sanitarios hacen referencia al apoyo y a la gestión directiva de los trabajadores de los centros de salud. También plantean la personalidad del trabajador como un elemento condicionante, lo que pone de manifiesto que la satisfacción de este colectivo está estrechamente vinculada con el soporte de las direcciones de recursos humanos de los centros de salud, hacia las necesidades concretas de cada uno de sus profesionales.

Otros estudios realizados en el ámbito de la APS en España ponen de manifiesto cuál es la percepción de las enfermeras respecto al apoyo de sus equipos directivos. Así, vemos que en un estudio realizado en Asturias ${ }^{30}$ para conocer la percepción que tenían las enfermeras del apoyo de la organización frente a sus necesidades laborales y personales, se constató que el grado de satisfacción percibida era de 4,78 sobre 10. Los resultados de otro estudio realizado en Baleares ${ }^{31}$ fueron similares, con valores no demasiado elevados y una percepción media de las enfermeras de 4,9 sobre 10 .

Queremos destacar que otros autores ${ }^{32,33}$ proponen aumentar la autoestima laboral y el nivel de satisfacción de los profesionales sanitarios, implicándolos en la toma de decisiones del equipo asistencial, potenciando su participación en la gestión, la elaboración de objetivos, guías clínicas, contratos programas, etc.

\section{Conclusiones}

Con estos referentes, resulta evidente que la actitud del personal directivo y de los responsables de Recursos Humanos de los centros de salud, hacia la mejora de la satisfacción de las enfermeras, será fundamental y es uno de los grandes retos a los que se enfrentan las direcciones de los centros asistenciales, siendo cada vez más conscientes del alto valor del capital humano como prestador de cuidados a la población ${ }^{34}$.

La insatisfacción en el trabajo puede resultar una amenaza para el desarrollo de las competencias profesionales, a la vez que puede reducir la capacidad de estas profesionales para detectar necesidades y establecer prioridades asistenciales.
Los cuidados de enfermería son fundamentales para el logro de los objetivos de salud, y no cabe duda de que son una de las piezas clave en el desarrollo de los sistemas de salud. Y no podemos olvidar que la excelencia profesional está muy vinculada al grado de satisfacción de sus profesionales.

\section{Bibliografía}

1. Gomez M, Sabeh EN. Calidad de vida. Evolución del concepto y su influencia en la investigación y la práctica. Instituto Universitario de Integración en la comunidad. Facultad de Psicología [acceso 19 marzo 2007]. Disponible en: http://www3.usual.es/inico/ investigacion/invesinico/calidad.htm

2. Caminal J. La medida de la satisfacción: un instrumento de participación de la población en la mejora de la calidad de los servicios sanitarios. Rev Cal Asistencial. 2001;16:276-9.

3. Peya M. Satisfacción laboral: una breve revisión bibliográfica. Nursing. 2008;26(2):56-9.

4. Spector PE. Job satisfaction, application, assessment, causes and consequences. London: SAGE; 1997.

5. Olivar C, González S, Martínez MM. Factores relacionados con la satisfacción laboral y el desgaste profesional de los médicos de Atención Primaria de Asturias. Aten Primaria. 1999;24(6):352-9

6. Salgado JF, Remeseiro C, Iglesias M. Clima organizacional y satisfacción laboral en una pyme. Psicothema. 1996;8(2):329-35.

7. Campbell L, Fowles ER, Weber BJ. Organizacional structure and job satsifaction in public Health Nursing Public Health Nurs. 2004;6:564-71

8. Brooks BA, Storfjell J, Omoike O, Ohlson S, Stemler I, Shaver J, et al. Assessing the quality of nursing work life. Nurs Adm Q. 2007;31(2):152-7.

9. Stamps PL. Nurses and work satisfacion. An Index for Measurement. AMER J Nurs. 1998;98(3):16kk1611.

10. Lu H, While AE, Barribal KL. Job satisfaction among nurses: a literature review. Int J Nur Stud. 2005;42:211-27.

11. Grau A, Suñer R, García M. Desgaste profesional en el personal sanitario y su relación con los factores personales y ambientales. Gac Sanit. 2005;19(6):46370

12. Aiken L, Clarke S, Slone D, Sochalski J, Busse R, Clarke H, et al. Nurses reports on hospital care in five countries. Health Aff. 2001;20(3):43-53.

13. Mittal V, Rosen J, Govind R, Degenholtz H, Shingala S, Hulland S, et al. Percetion gap in quality of life ratings: An empirical investigation of nursing home residents and caregivers. Gerontologist. 2007;47(2):159-68.

14. Caers R, Du Bois C, Gieter SD, Cooman RD Pepermans R. Measuring Community nurses job satisfaction: literature review. J Adv Nurs. 2008;62(5):521-9

15. Bégat I, Ellefsen B, Severinsson E. Nurses satisfactions with their work environment and the outcomes of clinical Nursing supervision on nurses experiences of well being a Norwegian study. J Nurs Manager. 2005;13:221-30.

16. Ponce J, Reyes H, Ponce G. Satisfacción laboral y calidad de atención de enfermería en una unidad médica de alta especialidad. Rev Enferm IMSS. 2006;14(2):65-73.
17. Andersen RM. Revisiting the behavior model and access to medical care: does it matter? J Health Soc Behav. 1995;36(1):1-10.

18. Hjortdahl P, Laerum E. Contnuity of care in general practice: effect on patient satisfaction. Br Med J. 1992;304:1287-90.

19. Fernández MI, Moinelo A, Villanueva A, Andrade C, Rivera M, Gómez MJ, et al. Satisfacción laboral de los profesionales de Atención Primaria del área 10 del Insalud de Madrid. Rev Esp Salud Pública. 2000;74(2):139-47.

20. Sánchez R, Alvarez R, Lorenzo S. Calidad de vida profesional de los trabajadores de Atención Primaria del Área 10 de Madrid. Medifam. 2003;13(4):291-6. 21. Peiró JM, Silla I, Sanz T, Rodríguez J, García JL. Satisfacción laboral de los profesionales de Atención Primaria. Psiquis. 2004;25(4):129-40.

22. Martínez D, Totterdell P, Alcocer CM, Colman D The source of display rules and their effects o Primary Health care professionals well-being. The Spanish J Psicol. 2009;12(2):618-31

23. Cortés JA, Martín J, Morente M, Caboblanco M, Garito J, Rodríguez A. Clima laboral en atención primaria: ¿Qué hay que mejorar? Aten Primaria. 2003;32(5):288-95

24. Caballero K. El concepto de satisfacción en el trabajo y su proyección en la enseñanza. Profesorado. Rev de currículum y formación del profesorado. 2002;6(1-2):1-10

25. Jeringan IE, Beggs J, Kohut G. Dimensions of work satisfaction as predictors of commitment type. J Manag Psychol. 2002;17(7):564-79.

26. García R, Lázaro MP, Quintana MA. Mejora de la calidad del trabajo enfermero en Atención Primaria tras una estrategia organizativa. Metas de Enferm. 2008;11(5):8-12.

27. Campbell SL, Fowles ER, Weber BJ. Organizacional structure and job satisfaction in Public Health Nursing. Public Health Nursing. 2004;21(6):564-71.

28. Ho WH, Chang SC, Shih YL, Liang RD. Effects of job rotation and role stress among nurses on job satisfaction and organizational commitment. BMC Health Serv Res. 2009;9(8):65-74.

29. Menárguez JF, Saturno PJ. Descripción del clima organizacional en equipos de atención primaria de una comunidad autónoma. Aten Primaria. 1999;23(5):280-4

30. Alonso M, Iglesias AI, Franco A. Percepción de la calidad de vida profesional en un área sanitaria de Asturias. Aten Primaria. 2002;30(8):483-9

31. Muñoz E, Coll JM, Torrent M, Linares L. Influencia del clima laboral en la satisfacción de los profesionales sanitarios. Aten Primaria. 2006;37(4):209-14

32. March JC, Oviedo E, Romero M, Prieto MA, Dane A. Mapa emocional de equipos de atención primaria en Andalucía. Aten Primaria. 2009;41(2):69-75.

33. Arriaga E, De la Torre J, Alberdi RM, Moreno J, García JM. La participación en la gestión como elemento de satisfacción de los profesionales: un análisis de la experiencia andaluza. Enfermería Global. 2003. [acceso 16 julio 2009]. Disponible en: http:// www.um.es/eglobal/

34. Mañas MA, Salvador C, Boada J, González E, Agulló E. La satisfacción y el bienestar psicológico como antecedentes del compromiso organizacional. Psicothema. 2007;19(3):395-400. 